\title{
Cultura de fluidez, estrategia para la rehabilitación y construcción de un ambiente sostenible
}

DOI: $10.20396 /$ labore.v13i0.8657905

Submetido 17 nov. 2019

Aceito 17 der. 2019.

Publicado 26 dez. 2019.

\author{
David Francisco Llamosa Escobar \\ $<$ https://orcid.org/0000-0002-8039-9730> \\ Universidad Nacional de Colombia / Bogotá [Colômbia]
}

RESUMEN

La naturaleza se caracteriza por ser un ente fluido y dinámico. Podemos establecer que los eventos cósmicos, los climáticos de la tierra, y los comportamientos biológicos, están regidos por fluidos fundamentales. Las formas fractales que evidenciamos en la naturaleza, son el resultado visible de la realidad vital y su dinámica constitutiva. Estas en un principio de economía natural, hacen posible que los fluidos se optimicen, permitiendo la creación de organismos. La fluidez natural contrasta con el estatismo de la geometría Euclidiana, y la parálisis propiciada por el diseño moderno, propios del entorno construido de las sociedades humanas, desde el inicio de la revolución industrial hasta nuestros días. El borde filoso disociativo, se ha opuesto de forma sistemática al borde poroso heterónomo, característico de los sistemas biológicos abiertos. Esto ha conllevado al deterioro del ambiente y a la segregación de las comunidades que pueblan la biósfera. Con el propósito de generar un ambiente sostenible, las operaciones en el entorno habitable, deberán fundamentarse en una consonancia con los flujos naturales, permitiendo construir con la naturaleza y no a pesar de ella. Una cultura de la fluidez permitirá políticas sostenibles, e intervenciones urbanas en el territorio, que superen los abismos culturales y sociales del presente.

PALABRAS CLAVE

Fluidez. Temporalidad. Fractal. Borde. Diseño. Sostenibilidad.

Fluidity culture, strateg̉y for rehabilitation and construction of a sustainable environment

\section{ABSTRACT}

Nature is distinguished as a fluid and dynamic entity. We can establish that the processes of displacement, climatic events of the earth, human and animal behavior, are governed by fundamental fluids. These fluids and their forms depending on the viscosity of time understood as the difference in time fluidity. The fractal shapes that we perceive in nature, are the visible result of their dynamics and intrinsic reality. The fractal shape enables the fluids, allowing the creation of organisms. This aspect contrast, essentially with the functionalism and Euclidean geometry, that has characterized the built environment, since the beginning of the industrial revolution. The dissociative cutting edge, it has opposed systematically to the natural porous flow, and the edge heteronomous, characteristic of open biological systems. To generate a sustainable environment and an alternative city, operations in the living environment, including the architectural and urban planning, must be conceived in consonance with natural flows.

\section{KEYWORDS}

Fluidity. Temporality. Fractal. Edge. Design. Sustainability. 


\section{Introducción}

La naturaleza se distingue por ser una entidad fluida y dinámica. Podemos establecer que los procesos de desplazamiento y formación de las galaxias, la creación de las estrellas, la formación del viento solar, los eventos climáticos de la tierra y el comportamiento humano y animal, se rigen por fluidos fundamentales. Dentro de una concepción materialista del mundo, anotaba Friedrich Engels en 1878: "Materia sin movimiento, es tan inconcebible como movimiento sin materia" (Engels, 1878, 1947, 1960). El movimiento es en consecuencia, inherente a la existencia material. Hay que anotar que el origen del dinamismo prevaleciente en la naturaleza y la forma general de los flujos, son explicados en términos de magnitudes diferenciales. Debido al fluido diferencial temporal, que podríamos llamar viscosidad del tiempo, los flujos son desiguales y permean toda la realidad material. Expresándose como la conjunción entre lo que fluye y lo que fluye más lentamente, como el contraste entre los tiempos largos y los tiempos cortos, como la confluencia de lo constante y lo variable, lo inerte y lo dinámico, la viscosidad temporal es responsable tanto del universo de los cambios como de las resistencias al cambio. Así, lo que se mueve y lo que es movido, lo que fluye y lo que genera fricción, deslizamiento y arrastre, conduce a la forma universal y a su diseño.

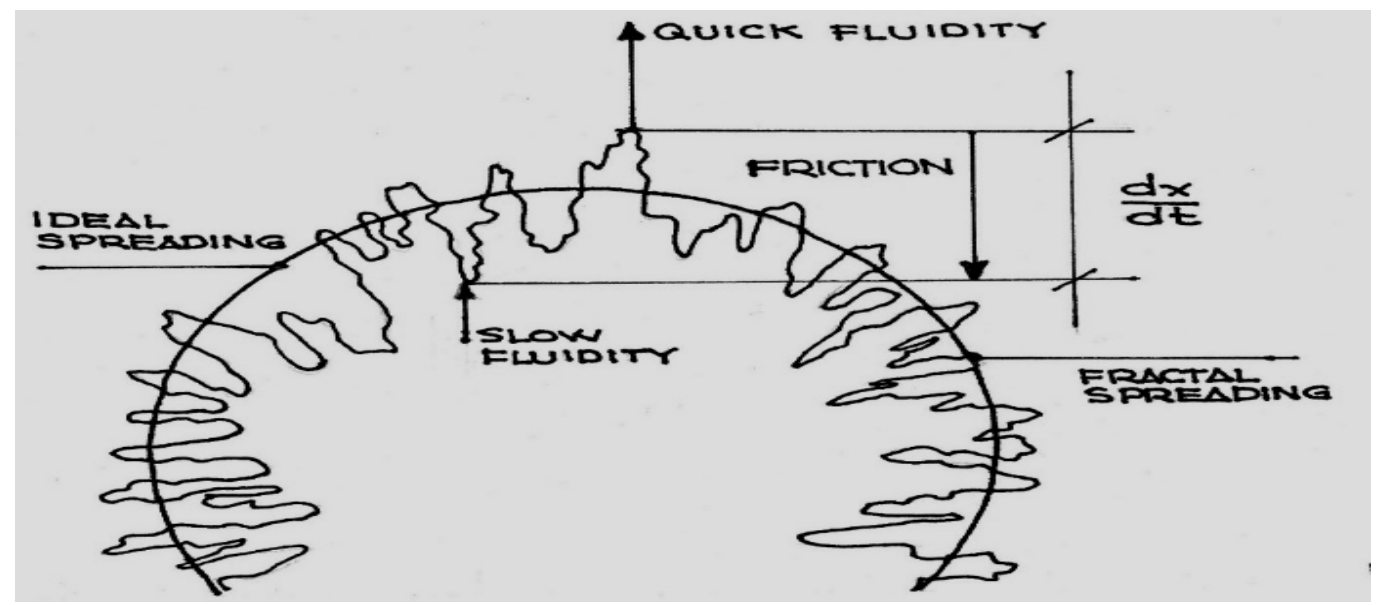

Figura 1. La Forma y el Diferencial.Fuente: Dibujo David Llamosa.

La viscosidad del tiempo o su flujo desigual, se debe fundamentalmente a la acción de la gravedad. La teoría general de la relatividad de Einstein, expone de forma magistral, la dependencia del flujo del tiempo debida a la acción de la gravedad. (Hawking 1992). La marcha del reloj se ve mermada por la intensidad de su campo. En la tierra, por ejemplo, en razón de su achatamiento, el reloj cerca de los polos, debido a su mayor cercanía al centro de masa, va más despacio que en el ecuador. Esta consideración, es la misma que explica la alteración del flujo del tiempo cerca de un campo gravitatorio intenso, como es el caso de los agujeros negros.

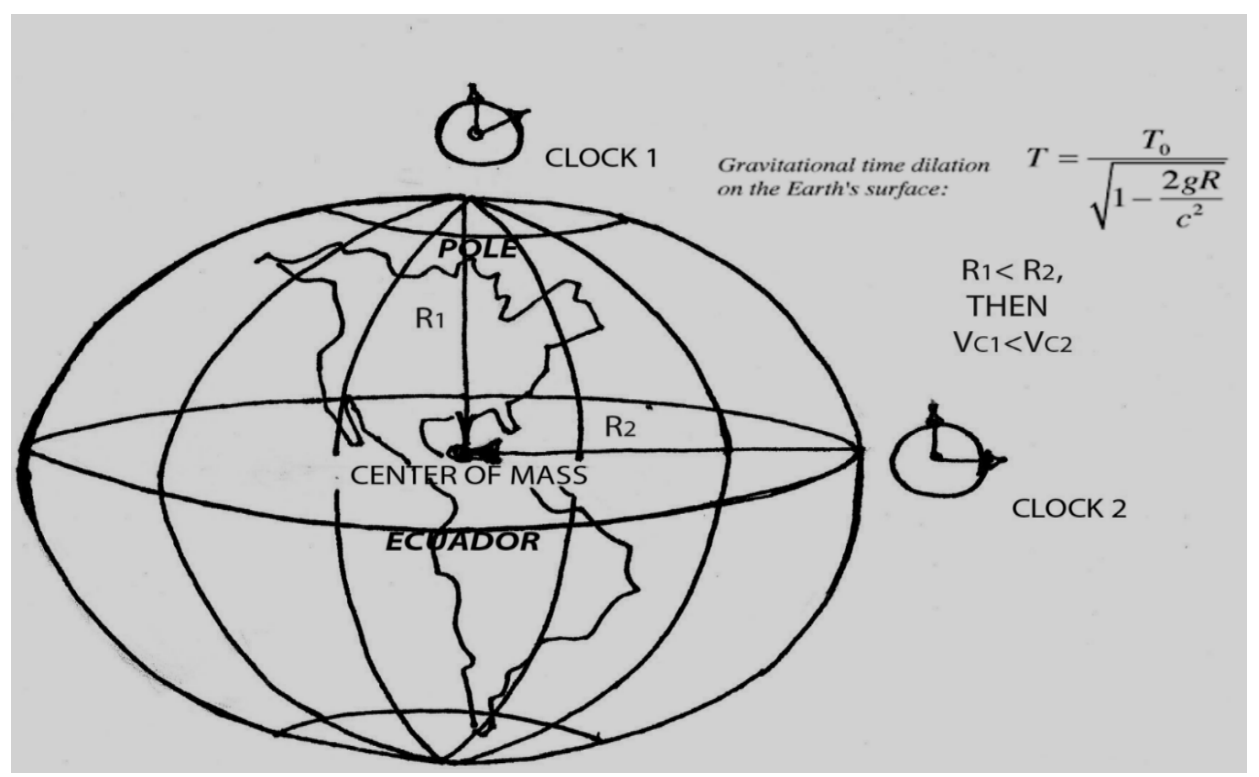

Figura 2. Dilatación del Tiempo. Fuente: Dibujo David Llamosa. 
Resultado de la expansión del universo, tenemos una dirección del tiempo y un proceso de hetero-génesis característico de la vida, en oposición al de homo-génesis, signado por la gravedad. (Davies, 1996). La diferencia en el flujo del tiempo puede explicar las formas de los flujos, el universo de las formas y la forma del universo. Si el tiempo fluyera de manera homogénea, el resultado sería el de un universo hipotético poblado de formas ideales. Si no hubiera cambio combinado con la oposición al cambio, la inercia no existiría, y tendríamos una materialidad ideal estática. Por supuesto que este universo solo podría existir en el ámbito de nuestras mentes. De ésta forma, el flujo del universo no es isócrono, y el espacio no es isótropo. Entendiendo esto podemos conciliar el tiempo corto de la partícula elemental, con el tiempo largo de las estrellas y galaxias, de los segundos con los eones. Los relojes de la realidad geológica, biológica e intraatómica, nos enseñan en la disparidad de sus ritmos, como se ha configurado la fisonomía heterogénea del ambiente planetario. La forma de todas las cosas, naturales y artificiales, son un resultado combinado de las acciones del tiempo largo en conjunción con las del tiempo corto. En el universo el tiempo se revela en la forma material, pero cada evento es único y se expresa mediante una forma particular.

Resultado del fenómeno contemporáneo de la temporalización del espacio, (el espacio clásico comprendía la espacialización del tiempo), son las heterocronías, o cuantificaciones diversas del tiempo, que en sus microritmos crean campos asociados. Campos, que no espacios, generados en la acción, pero nunca predeterminados por ella.

En efecto, es el tiempo mismo el que sufre también transformaciones sin precedentes, que afectan a nuestra propia conciencia en cuanto sujetos, siendo el tiempo lo que define el sentido interno, la identidad substancial a través de la sucesión de instantes. El punto temporal, como el punto geométrico no son nada, si no entran en relación con lo anterior y lo posterior, con el pasado y el futuro. El tiempo por tanto está vinculado al movimiento, que permite la sucesión de instantes, el paso fugaz a través de instantes nada, que se hacen algo cuando son recorridos (León, 1999).

El campo generado en el ámbito urbano, tal como sucede en la física, es el resultado de una dinámica. Puede entonces, la dinámica del sujeto, configurar en la ciudad, un pasado desfigurado, y un futuro que se potencializa en cada segundo. Hacen la ciudad, tanto los retro-futuros, los futuros-presentes, y los pretéritos-futuros. Es el reino de las cronotopias o de los tiempos hechos lugar.

\section{Los fractales}

Los flujos en la naturaleza son cíclicos. Flujo y reflujo han modelado la faz de la tierra. Es esta periodicidad la que ha permitido el desarrollo de la vida y el incremento de la diversidad biológica. Habrá que notar que el curso de éstos flujos es muy particular. Como resultado de aceleraciones y fuerzas, configuran el lugar, eligiendo un camino crítico. Éste curso ha sido identificado como la forma fractal. Las formas fractales son mucho más que las formas caprichosas que podríamos encontrar en la naturaleza. Permitiendo el desarrollo de la vida, son el resultado visible de una realidad intrínseca y de una dinámica constitutiva material. A partir de la ínfima súper cuerda, o la cadena de $\mathrm{ADN}$, hasta las galaxias y los quásares, se podría aplicar el principio de iteración que rige el fenómeno fractal. Este se explica como la similitud formal que poseen las partes y el todo de los objetos, Repetido en principio $n$ veces a escalas diferentes, es por definición, el objeto fractal, recursivo y auto-similar. La forma fractal permite que los fluidos se optimicen, favoreciendo la creación de organismos. Se puede determinar claramente que, desde la amplia geografía, hasta los minúsculos sistemas biológicos, la forma fractal es la facilitadora de la organicidad del mundo. En la obtención de formas, la naturaleza no escatima esfuerzos para ahorrar energía, maximizar el espacio, y fomentar el intercambio de los soportes de la vida. Creemos también que, a través de su multiplicidad formal, la fractalidad, hace arte en la naturaleza, crea su belleza y encanto característicos (Peitgen \& Richter, 1986). Pero es también en el contexto urbano, donde la forma fractal y su iteración característica, se demuestra de forma reveladora:

"Por la propia esencia de la ciudad, la disciplina que mayor influencia ha ejercido sobre la visión organicista ha sido la nueva geometría. Desde un principio se intuyó que los fractales podrían ser operativos en el análisis de muchos fenómenos urbanos típicamente contemporáneos: el dífuminado de los bordes, la porosidad del tejido, la capilaridad del viario, etc. El concepto de scaling, propuesto por Peter Eisenman, (Eiseman 1988), se basaba en la suposición de que todas las escalas de la arquitectura (desde la puerta, a la habitación, el apartamento y el edificio) y todas las escalas de la ciudad (desde el edificio, a la calle, el barrio y la autopista) son la misma, pues son múltiplos de las dimensiones del cuerpo humano. Si se usa como generador de las formas urbanas y arquitectónicas, el scaling se convierte en una unidad de medida universal que es aplicable a todas las componentes de la ciudad. Es este modo, el principio de la autosimilitud se extiende a la ciudad” (García Vásquez 2006). 


\section{Labor \& Engenho}

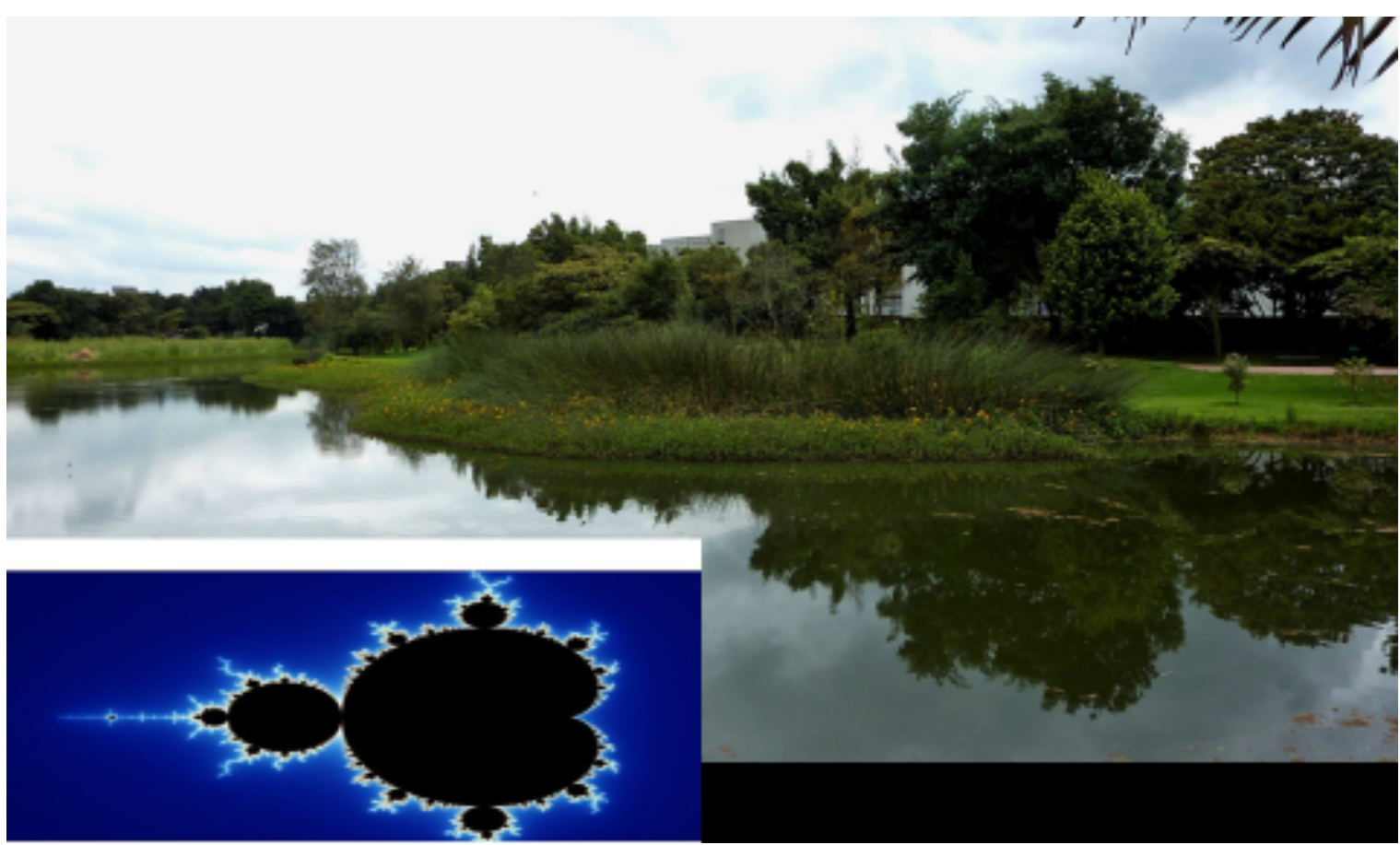

Figura 3. La Forma Fractal y el Conjunto de Mandelbröt. Fuente: Foto David Llamosa.

Los eventos complejos de características dinámicas asociadas al fractal, tales como el clima, la economía, o las alteraciones geológicas, se han podido modelizar mediante los atractores de Loren\%. Estos constituyen simulacros matemáticos que dan cuenta de los procesos no-lineales y de naturaleza caótica que ocurren en la naturaleza. Hay que anotar que la vida de las ciudades, se debate entre las turbulencias del clima, en conjunto con las de la economía y las transformaciones sociales.

Los extraños atractores demostraron su operatividad a la hora de medir manifestaciones como las turbulencias, expresiones naturales hasta entonces inabordables por su altísimo grado de desorganización e inestabilidad. Cuando los ordenadores permitieron representar los cambios de fase de un sistema, los extraños atractores empezaron a aparecer por doquier: en los flujos de los fluidos, en los remolinos, en las llamas, etc., lo que significaba que, al Igual que su forma, también el funcionamiento de los fenómenos naturales de apariencia caótica se podría reducir a patrones abstractos, matemáticos y universales, Se abría paso, así, la segunda gran conclusión aportada por las ciencias del caos; existe orden en el caos (García Vásquez, 2006).

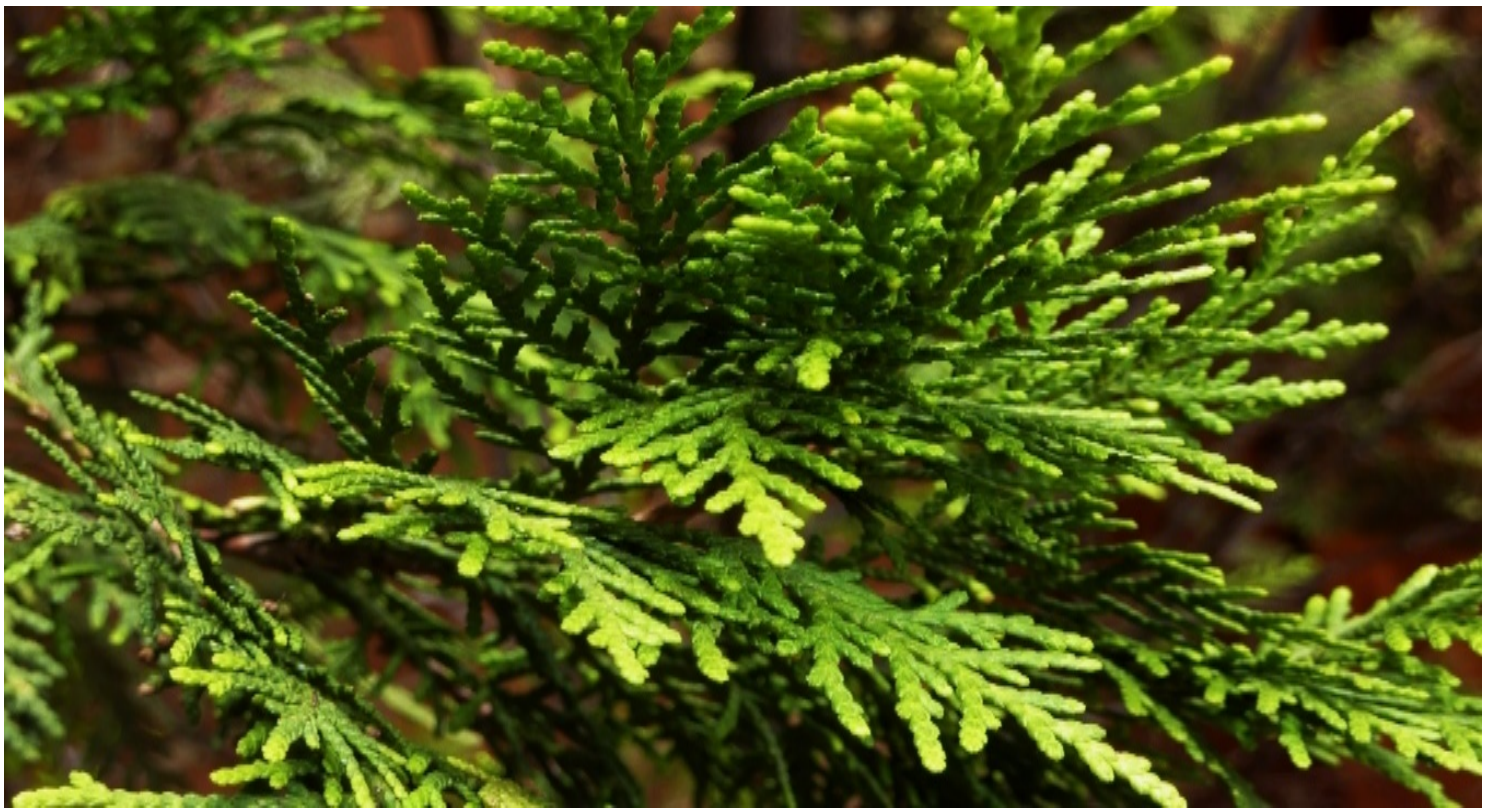

Figura 4. La Naturaleza hace Arte mediante la Forma Fractal. Fuente: Foto David Llamosa. 
Los atractores y la lógica del caos, entonces, bien pueden asociarse al fenómeno urbano, sobre todo cuando las ciudades demuestran ser especialmente vulnerables a las variaciones mínimas que se experimentan en el contexto global, (Efecto mariposa), y cuando de forma recíproca, el contexto del globo se ve afectado por el impacto de las grandes ciudades. Se infiere así que, los flujos y la fluidez, comprenden un aspecto fenoménico y un aspecto formal. El fenoménico tiene que ver con el caos y la disparidad del flujo temporal, y el formal con la fractalidad asociada.

\section{La ciudad y los flujos}

Es un ejemplo muy diciente, cómo el flujo del tiempo se aprecia desigual en la configuración de la ciudad. Los estratos culturales de período largo, coinciden en simultaneidad con los de período corto, e inclusive con los más vertiginosos. Aldo Rossi en su Autobiografía científica afirmaba: "El doble significado atmosférico y cronológico del tiempo, es el principio que rige toda construcción. La conjunción de estos dos tiempos revela simultáneamente lo breve y lo perdurable que poseen las formas en arquitectura" (Rossi, 2000). La tradición y la innovación, así representadas, en contradicción dialéctica conforman, el dinamismo responsable del desarrollo histórico.

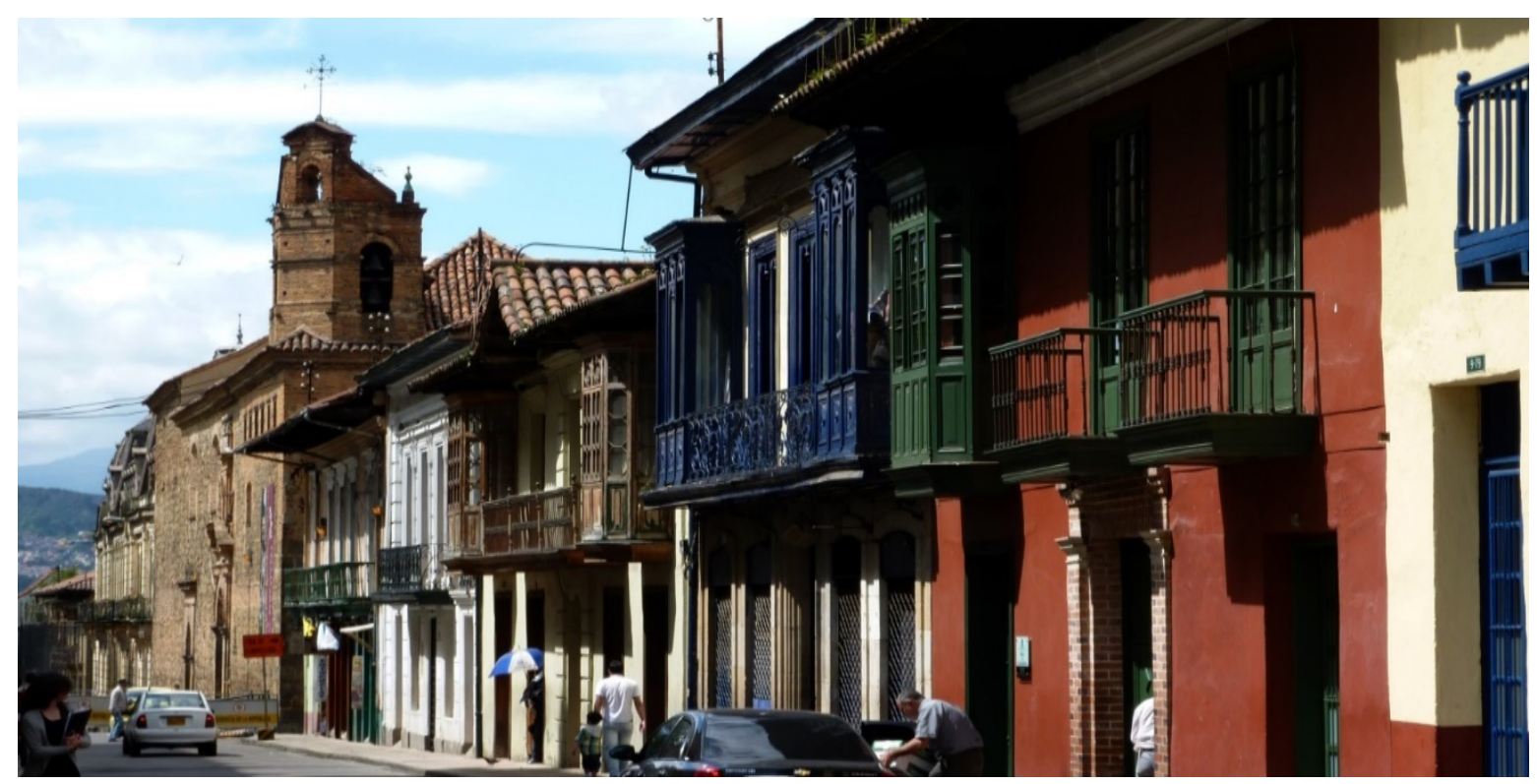

Figura 5. La Ciudad y el Tiempo Largo. Centro Histórico de Bogotá. Fuente: Foto David Llamosa.

En la definición de la arquitectura, se recurre con frecuencia a los tipos y a las formas. Los tipos representan las fuerzas conservadoras de carácter constante, y las formas, la variabilidad y el cambio. Aplicando el principio de causalidad, podríamos predecir el tipo, pero no la singularidad de la forma. Esta singularidad resulta impredecible, pero conjuntamente con el tipo, constituye el texto donde se lee y consigna la historia de la ciudad. Los tipos y las formas no son otra cosa que la expresión en la cultura de fuerzas naturales subyacentes. La memoria podría considerarse en la ciudad, como el acto de resistencia, que se opone a la dominancia de lo volátil y lo efímero, representada en el cambio.

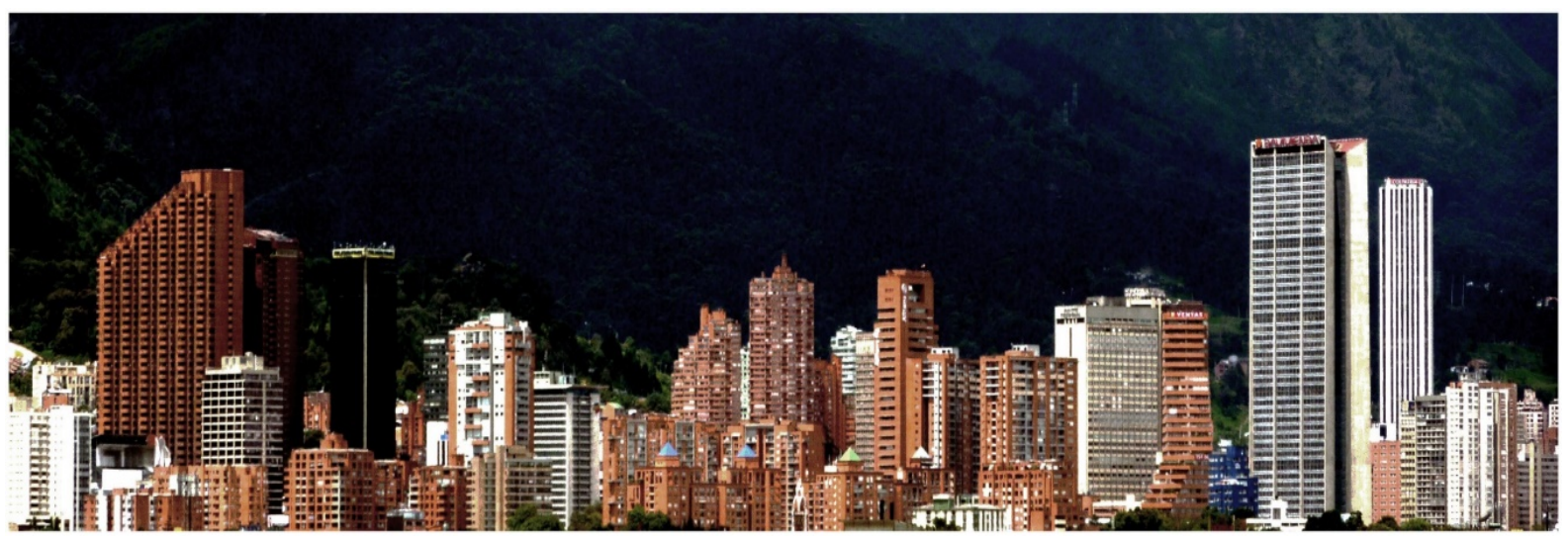

Figura 6. La Ciudad y el Tiempo Corto. Centro Internacional en Bogotá. Fuente: Foto David Llamosa. 
En un propósito idealista de estabilidad y equilibrio, las sociedades humanas han buscado refugiarse del caos natural, mediante el orden representado en la construcción artificial. Pero la verdad es que, debido a la fluidez y la perpetuidad del movimiento, (nada en la naturaleza es geométrico y estático, en el sentido ideal del término), es imposible lograr la estabilidad o completitud deseada. Las formas ideales de la arquitectura y de la ciudad, representativas de lo inerte, particularmente las propuestas en la revolución modernista ortodoxa y el estilo internacional, están negativamente predispuestas al cambio y a la fluidez. Obstruyendo de forma sistemática, a escala geográfica, los flujos naturales y sociales, han contribuido de forma evidente al deterioro ambiental y social que padecemos hoy en la contemporaneidad.

Mientras que la ciudad del XIX desubstancializó el espacio natural, la ciudad del final del XX ha hecho desaparecer el espacio geográfico, por lo que puede decirse que no es esta la época del fin de la historia como anunció Fukuyama, sino la del fin de la geografía. Pues la ciudad post-moderna ha contemplado la eliminación de los últimos vestigios de las funciones sociales, que aún conservaban las buellas de los lugares naturales de las polis (León, 1999).

\section{El borde}

El diseño prismático de inspiración Euclidiana, ha signado el entorno construido desde los inicios de la modernidad. La arista y el borde excluyente del filo, se ha opuesto sistemáticamente al borde heterónomo, poroso del fractal, característico de los sistemas biológicos abiertos. La cristalización inerte del diseño funcionalista, y la parálisis asociada del lindero y la frontera, han provocado una fractura en la organicidad del territorio, contribuyendo de forma visible al desequilibrio ambiental, y a la alteración negativa del comportamiento de los seres vivos en su hábitat (Llamosa Escobar, 2013). Es dable considerar que la planificación y jurisdicción del territorio, va en la mayoría de los casos, en contravía de la naturaleza del territorio, pero también de la naturaleza de las sociedades y de los grupos humanos. Así como el mundo externo que percibimos, está en una relación íntima con la percepción que poseemos de nosotros mismos, los organismos requieren para su sobrevivencia, además de su fisiología interna, de una relación efectiva con su entorno. La lección para un ordenamiento del territorio es que, individuos, colectividad y ambiente, deben conformar una unidad orgánica. Una ciudad segregada es una ciudad injusta, insana, no sustentable, y es en su desavenencia, antiestética... Debemos construir como dijo Newton, no muros o cercas, sino puentes que permitan el movimiento de la vida.

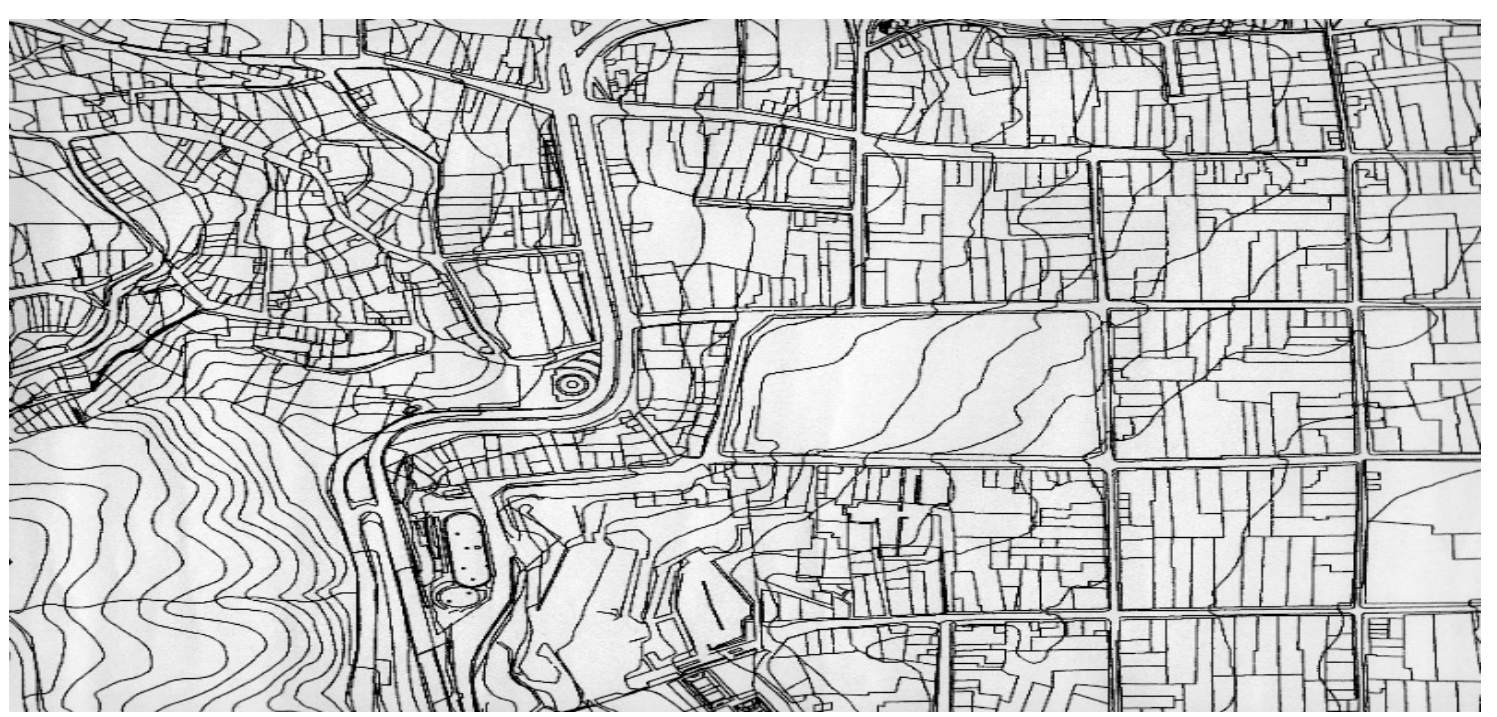

Figura 7. Damero Español en contraste con la geografía. Fuente: Plano de Santa Fé de Bogotá.

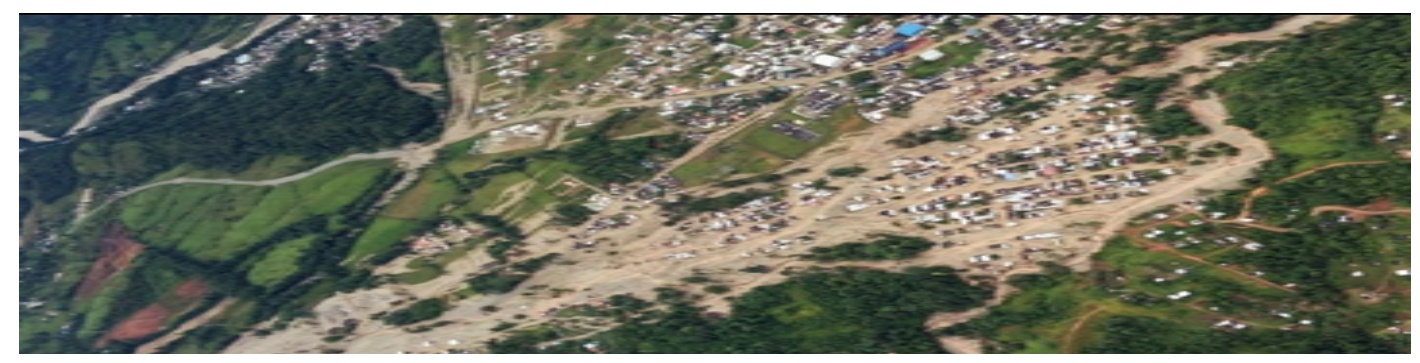

Figura 8. Las Ciudades Obstruyen con Frecuencia los Flujos Naturales. Fuente: Ciudad de Mocoa en Colombia. 


\section{Labor \& Engenho}
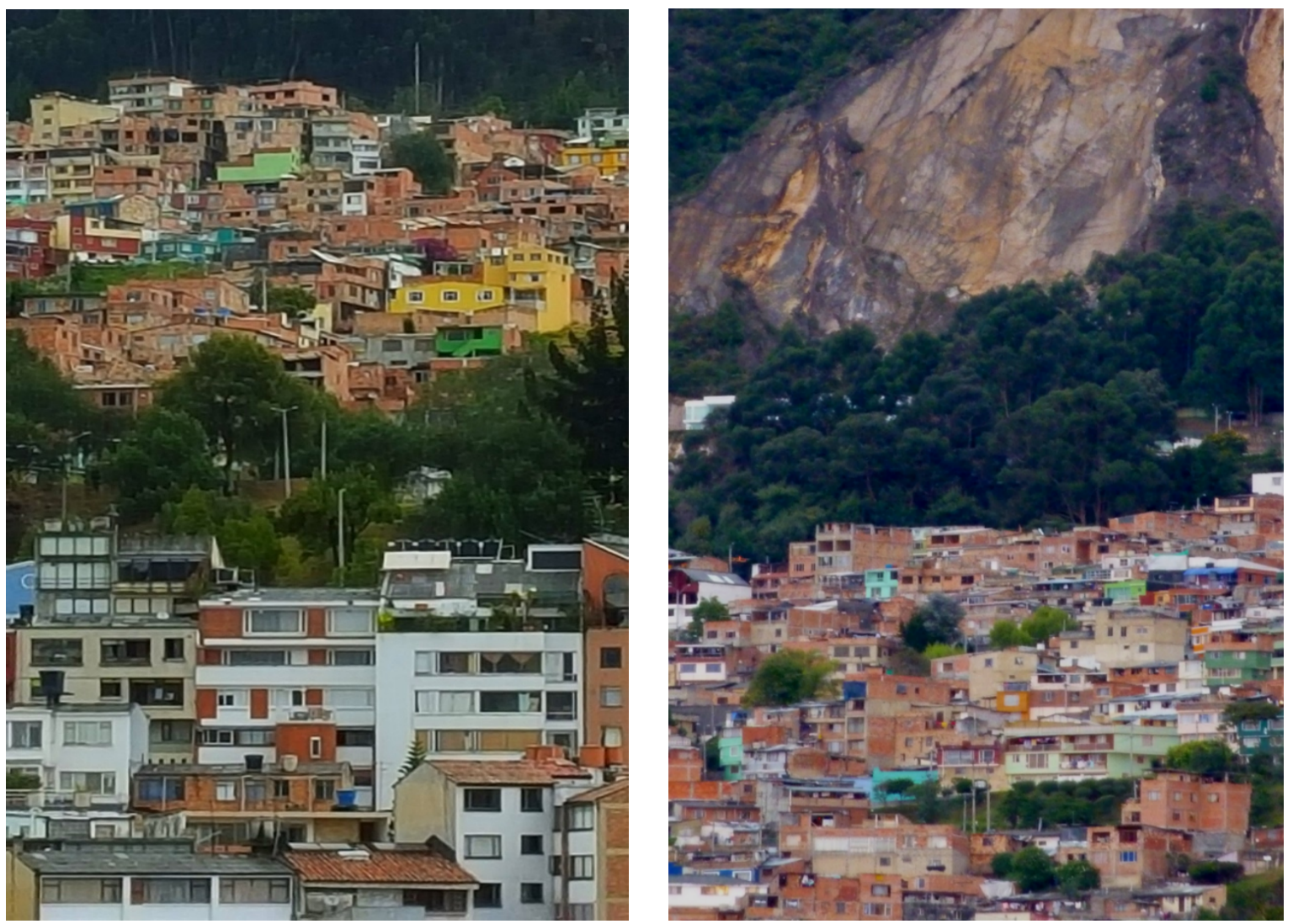

Figuras 9a e 9b. Bordes en Bogotá. Fuente: Fotos David Llamosa.

\section{Cultura de la fluidez}

Todo cambia, todo deja su sitio y fluye...

(Eurípides c. 416 a de C.).

La misma ciudad, el mismo organismo electrófago y depredador, causante del cambio climático, es hoy en día el hábitat más vulnerable. La ciudad se ha constituido en términos de su impacto en la biosfera, en la peor antítesis de la ciudad. Para generar un ambiente sostenible y concebir una ciudad alternativa, las intervenciones en el entorno de la vida, incluida la arquitectura y la planificación urbana, deben estar en sintonía con los flujos naturales. Las operaciones en el entorno construido, deben apuntar a una recuperación de la fluidez para mejorar las relaciones entre la naturaleza y el interés humano. Se debe estar consciente de que se han construido las urbes en medio de un torrente de fluidos. Una filosofía del fluido, que no de la movilidad, es lo que nos permitiría construir con la naturaleza. Una cultura de la fluidez, permitiría políticas sostenibles que superen los abismos culturales y sociales, fortalezcan el tejido social y favorezcan la continuidad de la vida.

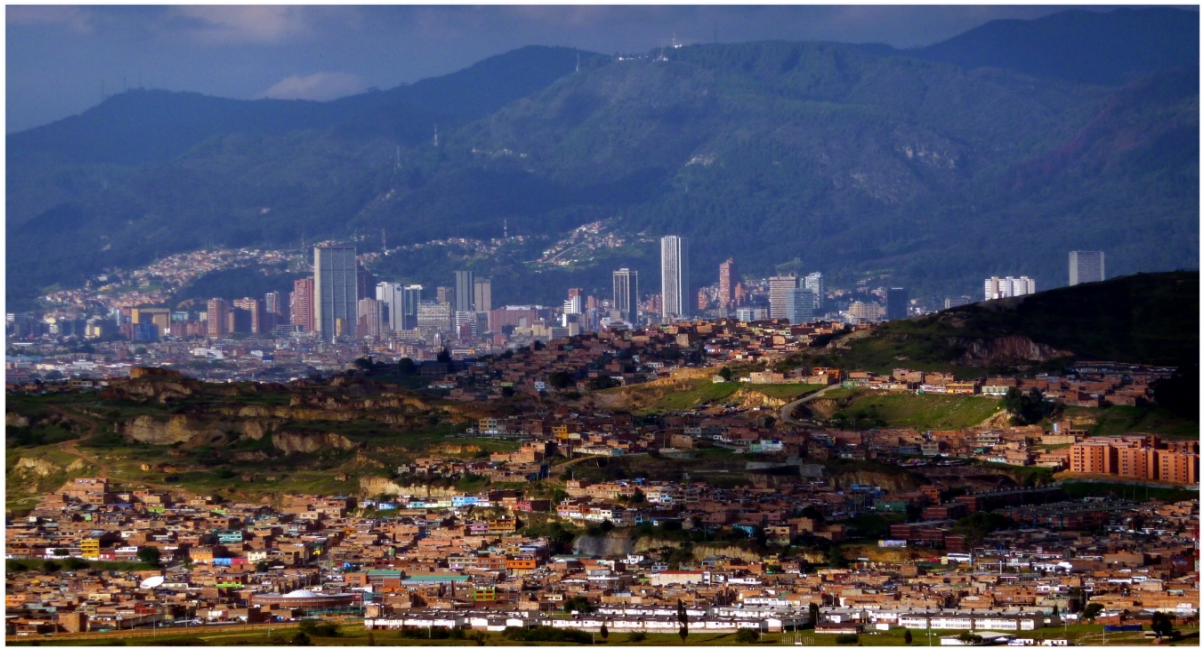

Figura 10. Bogotá, vista del

Centro Financiero desde

"Ciudad Bolívar".

Fuente: Foto David Llamosa. 
El espacio de la arquitectura y la ciudad, sigue siendo una entidad determinada por la historia, la naturaleza y el comportamiento humano. De ésta forma, el argumento lógico de la arquitectura no debe provenir de una geometría pura o de consideraciones abstractas externas a la vida. Una teoría del hábitat, debe derivarse de la naturaleza y de la práctica social y cultural. La única lógica que sería aplicable, tanto a la arquitectura como a las ciudades, es la del universo biológico subyacente. La referencia entonces debe ser el caos y las formas, que gobiernan la vida. Esto desde luego, no supone lo biológico derivado del darwinismo o del viejo mecanicismo, que entendía la arquitectura como una contradicción entre el organicismo y el funcionalismo, sino de los sistemas caóticos y de la complejidad de las realidades discretas.

Donde comienza el caos acaba la ciencia clásica. Durante todo el tiempo en que el mundo ha tenido a físicos cuestionándose sobre las leyes de la naturaleza, ha habido una especial Ignorancia hacia el desorden de la atmósfera, de las turbulencias marinas, de las fluctuaciones de las poblaciones de animales salvajes, de las oscilaciones del corazón y el cerebro. La parte irregular de la naturaleza, la parte discontinua y errática, siempre ba sido un misterio para la ciencia o, aún peor; una monstruosidad (Gleick, 1987).

La ciencia contemporánea, se ha llegado a fundamentar en la física biológica. La visión cosmológica actual, está comprometida en demostrar el cosmos como una entidad esencialmente macro-biológica, donde la consciencia desempeña un papel fundamental en la construcción de la realidad. Invirtiendo los términos clásicos, el universo no procura la vida, sino es ésta la que lo crea (Lanza \& Berman, 2010).

Mediante una planificación urbana inteligente, que asuma de modo importante los flujos naturales, es posible conciliar la naturaleza de la naturaleza, con la naturaleza humana. La propuesta, entonces consistiría en un urbanismo dinámico, que contraste con el urbanismo estático e introvertido de la tradición modernista. Este nuevo urbanismo fomentaría el crecimiento y el desarrollo de la economía, pero sobre todo el restablecimiento de los flujos naturales, garantizando la disolución del borde disociativo, la continuidad de los bio-sistemas, la equidad, la participación, y la integración del cuerpo social. Un urbanismo dinámico así concebido, implica entonces un urbanismo sostenible.

Destacamos que el arquitecto en la actualidad debe construir en lo construido, en ciudades con historia, debe construir en lugares naturales, donde reinan las leyes naturales. Debe entender que el ámbito urbano ha trascendido la esfera local de la geografía para adentrarse en la planetaria. Cómo se podría creer hoy en la inteligencia de una urbanía que, en su pedantería formal y ostentación tecnológica, ignore el hábitat natural dentro del cual está inmersa. La creación del hábitat en las ciudades implica no solo aspectos de diseño, o estrategias de intervención, sino también el estudio de las ciencias naturales y un especial respeto por el patrimonio natural, humano, y los valores sociales. El planificador del presente debe en consecuencia, renovar fundamentalmente los métodos y los modos de pensar la ciudad.

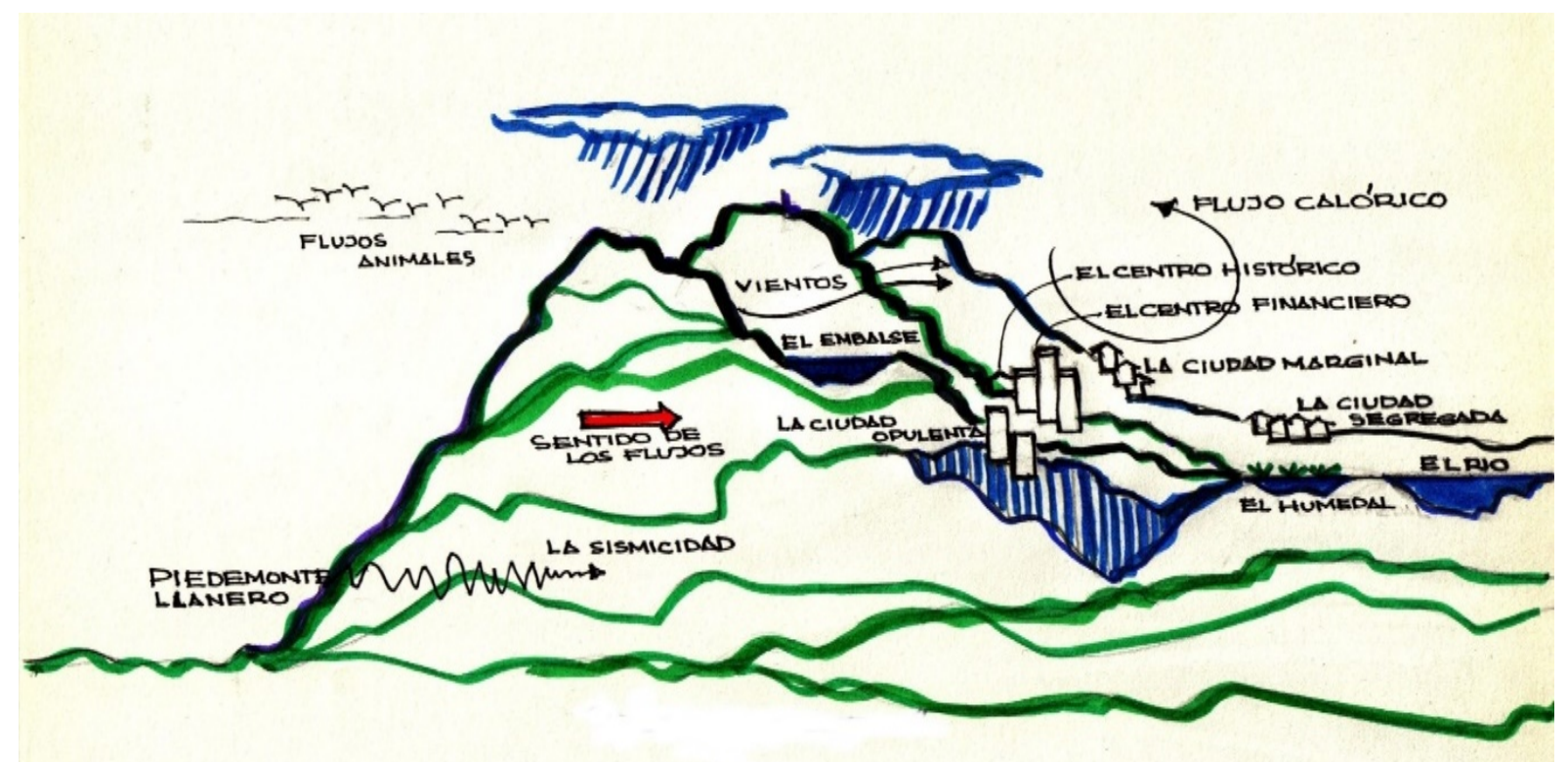

Figura 11. Bogotá y los flujos. Fuente: Dibujo David Llamosa. 
Las estrategias sostenibles, derivadas de una cultura de la fluidez, se podrían resumir en las siguientes acciones:

1. Estudiar la historia de los flujos naturales y humanos en los lugares donde se establecen, donde se han establecido y, donde se establecerán los asentamientos humanos.

2. Establecer como prioridad la recuperación de las cuencas de los ríos, los humedales y los recursos hídricos en general.

3. Disolver el borde filoso en favor del borde poroso. Para establecer una comunicación entre los bio-sistemas y los entes sociales, los límites políticos y físicos deben permitir los flujos animales y humanos.

4. Permitir la funcionalidad orgánica. Esto significa otorgar facilidades para el intercambio de energía y fluidez a los soportes de la vida.

5. Tener en cuenta los fenómenos fractales en términos de la funcionalidad natural, y el comportamiento de los seres vivos. Los fenómenos fractales, son una guía para un buen urbanismo, más no constituyen en el diseño, un repertorio obligado de formas aplicables.

6. Crear una red entre la naturaleza y el espacio público, los flujos y la movilidad, afianzando los valores intrínsecos del paisaje natural y su relación con el paisaje urbano.

7. Conciliar, el flujo lento de los lugares y los edificios históricos, con el flujo rápido característico de las nuevas ciudades.

8. Construir ciudades a una escala adecuada, donde la fauna y la flora, los sistemas hídricos, las condiciones geotérmicas, la fluidez calórica, el riesgo sísmico, las fluctuaciones de la humedad, los vientos predominantes y la iluminación característica del sitio, sean entendidas como contexto.

9. Reestablecer en lo posible la permeabilidad del suelo urbano. El suelo es la piel de la tierra que alberga vida, con un natural fluido e intercambio.

10. Considerar urgente y necesario, que todo plan de movilidad urbano incluya como prioridad, los flujos naturales sobre los artificiales, evitando así posibles obstrucciones.

11. Descubrir las intersecciones críticas que existen, entre los flujos naturales y las construcciones artificiales, para determinar las acciones de recuperación y rehabilitación a seguir.

12. Implicar en la estrategia de la fluidez, acciones de mitigación, recuperación y de planificación inteligente. Esto comprende una relación inédita entre el pasado y el futuro de la ciudad.

\section{Conclusión}

Del tiempo universal se derivan la multiplicidad de las formas universales. La forma natural es el resultado del fluido diferencial, viscoso, del tiempo. El tiempo se hace visible, no mediante el espacio, sino mediante la forma fractal. Las formas biológicas en particular, lo enseña la ciencia, están modeladas por una direccionalidad del tiempo característica. Las formas creadas por el hombre no deben ser un símil, sino un eco inteligente de las formas naturales. La base científica de nuestra estrategia de diseño sostenible, es una consideración de la física y la geografía como punto de partida, claramente vinculado con el tiempo largo, la naturaleza de los lugares, la memoria y el patrimonio. La incorporación de la ciencia al diseño, es la física combinada con la geografía, la biología y la historia.

El más importante argumento derivado del trabajo del físico Stephen Hawking, es que la creación de la realidad es la creación del tiempo. Sin tiempo no habrían formas, no habría atmósferas ni lugares, no habría historia. ¿Cómo podríamos explicar el fenómeno de las ciudades sin el flujo del tiempo? Dice la Carta de Megaride: "La ciudad del siglo XXI, la ciudad cableada, la ciudad de la paz y la ciencia, debe ser la expresión de la historia urbana y la cultura consolidadas por el flujo del tiempo" (Carta de Megaride 1995). Pensemos el planeta Tierra y la ciudad, en la era del tiempo. La naturaleza sólo entrega futuro a quien la sabe pensar. En esto se resume el argumento filosófico de nuestra propuesta.

\section{Referencias}

Burrough, P. (1981). Fractal dimensions of landscapes and other environmental data. Nature 294.

Bauman. (2000). Liquid modernity. Cambridge : Polity Press.

Carta de Megaride. Citta della pace, citta della scienza. (1995). Napoli : Universita degli studi di Napoli "Federico II".

Coleman, P., \& Pietronero, L. (1992). The fractal structure of the universe. Phys. Rep. 213.

Davies, P. (1996). Sobre el tiempo. Barcelona : Crítica. 


\section{Labor \& Engenho}

Eisenman, P. (1988). Moving Arrows, Eros and Other Errors. Arquitectura, 270, Madrid.

Engels, F. (1878). Herrn Eugen Dührings Umwälzung der Wissenschaft. Leipzig.

Engels, F. (1947). Anti-Dübring. Herr Eugen Dühring's Revolution in Science. Progress Publishers.

Engels, F. (1960). Anti-Düring. Montevideo : Ed. Pueblos Unidos.

Gleick, J. (1987). Chaos. Making a New Science. New York : Penguin Books. (versión castellana: Caos. La creación de una ciencia. Barcelona : Seix.Barral, 1998).

García Vásquez, C. (2006). Ciudad Hojaldre, visiones urbanas del siglo XXI. Barcelona : G.G.

Hawking, S. (1992). Historia del tiempo. Barcelona : Planeta Agostini.

Lanza, R., \& Berman, B. (2010). Biocentrism. Dallas : Benbella books.

León, F. (1999). Metápolis, la ciudad deconstruida. Revista Astrágalo, 9. Madrid.

Llamosa Escobar, D. F. (2015). El complejo temporal de la ciudad. Labor \& Engenho. 9(3) pp.58-73, jul./set. Unicamp. Campinas [SP] Brasil.

Llamosa Escobar, D. F. (2013). Más allá del borde, un reto para el mundo. Revista Hito, 27. Bogotá.

Llamosa Escobar, D. F. (2011). Acerca del acontecimiento. Revista Nodo. UAN. 6. Bogotá.

Lynn, G. (1998). Folds, Sodies \& Blobs : collected essays. Bruselas : La Lettre Volee.

Mandelbrot, B. B. (1982). The fractal geometry of nature. San Francisco : W. H. Freeman \& co.

Peitgen, H., \& Richter, P. (1986). The beauty of fractals. Heidelberg: SpringerVerlag.

Pope, A. (1996). Ladders, Architecture at Rice. New York : Princeton Architectural Press Houston.

Rogers, R. (2010). Ciudades para un pequeño planeta. Barcelona : G.G.

Rossi, A. (2000). Autobiografia Cientifica. Barcelona : G.G. 